\title{
Physician Empathy and Diabetes Outcomes
}

\author{
Alexander Chaitoff, MD, MPH', Michael B. Rothberg, MD, MPH', and Kathryn \\ A. Martinez, $P h D, M P H^{2}$
}

${ }^{1}$ Cleveland Clinic Lerner College of Medicine, Case Western Reserve University, Cleveland, USA; ${ }^{2}$ Center for Value-Based Care Research , Cleveland Clinic, Cleveland, OH, USA.

J Gen Intern Med 34(10): 1967

DOI: $10.1007 / \mathrm{s} 11606-019-05188-\mathrm{Z}$

(C) Society of General Internal Medicine 2019

W e appreciate the response from Hojat and colleagues regarding our study. In our manuscript, we extensively outlined reasons why we might not have been able to replicate the original study's findings. Those that the authors proposed in their letter, however, are unlikely causes.

They propose our findings may have differed due to the mandatory communication trainings at Cleveland Clinic. Yet our methods stated the Jefferson Scale of Empathy (JSE) scores used in our study were collected prior to training exposure. It's true we had a larger proportion of Internal versus Family Medicine physicians and a moderately different case mix of patients, but our finding of no association between empathy and diabetes outcomes was consistent in all multivariable and sensitivity analyses. Whether using their empathy cut points, analyzing scores continuously to maximize statistical power, or looking at subsets of patients with poorly controlled diabetes, we found no relationship between JSE and outcomes.

As described in detail in our paper, our inability to reproduce the original study's conclusions is not unusual - research findings are often reversed. ${ }^{1}$ This is why it's important to replicate studies. It may be true that empathy was associated with diabetic control among patients of 29 Family Medicine physicians at Thomas Jefferson $>10$ years ago, but their conclusion that "physician empathy is an important factor associated with clinical competence and patient outcomes" is not true now, at least not at the Cleveland Clinic.

Finally, the authors cite our "error in judgment" for acknowledging that the two studies that found correlations between empathy and diabetes outcomes were conducted by the same group. We fear they felt we were impugning their integrity, which is not the case. Research shows even when studying the same data, different researchers often come to different conclusions, ${ }^{2}$ highlighting the benefit of having multiple groups independently study similar topics. Additionally, financial interests have been shown to lead to unconscious biases, ${ }^{3}$ and investigators that are professionally invested in a specific field may be more likely to see associations in data than impartial methodologists. ${ }^{4}$ These implicit phenomena are reasons that authors are required to disclose financial conflicts of interest, and less commonly, professional ones. We have no specific stake in the outcome of this study. We hope that others will also attempt to replicate this work and add to our knowledge about the impact of empathy on patient outcomes.

Corresponding Author: Kathryn A. Martinez, PhD, MPH; Center for Value-Based Care Research Cleveland Clinic, Cleveland, OH, USA (e-mail: MARTINK12@ccf.org).

\section{Compliance with Ethical Standards:}

Conflict of Interest: The authors declare that they do not have a conflict of interest.

\section{REFERENCES}

1. Ioannidis JPA, Chen J, Kodell R, Haug C, Hoey J. Why Most Published Research Findings Are False. PLoS Med. 2005;2(8):e124. https://doi.org/ 10.1371/journal.pmed.0020124.

2. Silberzahn R, Uhlmann EL, Martin DP, et al. Many analysts, one data set: Making transparent how variations in analytic choices affect results. Adv Methods Pract Psychol Sci. 2018;1(3):337-356.

3. Dana J. Conflict of Interest in Medical Research, Education, and Practice. Vol (Field MJ, Lo B, eds.). Washington DC: National Academies Press; 2009.

4. Panagiotou OA, Ioannidis JPA. Primary study authors of significant studies are more likely to believe that a strong association exists in a heterogeneous meta-analysis compared with methodologists. J Clin Epidemiol. 2012;65(7):740-747.

Publisher's Note Springer Nature remains neutral with regard to jurisdictional claims in published maps and institutional affiliations. 